\title{
ARTICLE
}

\section{Development of radioactivity calculation method for nuclear power plant with radioactivity database}

\author{
Masahiko Kurosawa $^{a^{*}, K}$ Kaoru Matsushita ${ }^{a}$, Satoshi Yagishita ${ }^{a}$, Takayuki Hirouchi ${ }^{a}$, \\ Hidehiko Iida $^{b}$, Tatsuya Ikeda ${ }^{b}$, Koh Akatsu $^{\mathrm{b}}$ and Ken-ichi Tanaka ${ }^{\mathrm{c}}$ \\ ${ }^{a}$ Toshiba Corporation, 8, Shinsugita-Cho, Isogo-ku, Yokohama, 235-8523, Japan; ${ }^{b}$ Toshiba Plant Systems \& Services Corporation, 8 , \\ Shinsugita-Cho, Isogo-ku, Yokohama, 235-8523, Japan; ${ }^{c}$ The Japan Atomic Power Company, 1-1, Kanda-Mitoshiro-cho, \\ Chiyoda-ku, Tokyo, 101-0053, Japan
}

\begin{abstract}
A new calculation method for radioactivity in a nuclear power plant is proposed. It can calculate radioactivity in shorter time than the conventional method with the same accuracy. The characteristic of the method is the effective usage of the radioactivity database applicable to the whole region of the plant. The database is provided for typical neutron irradiation conditions, cooling times and elemental compositions in a nuclear power plant by calculation with an isotope generation and depletion code such as ORIGEN-S. The effects of the method on both the time reduction and the calculation accuracy have been confirmed through application to an existing BWR in Japan. The calculation time can be shortened to around $1 / 4000$ of the conventional method for a typical case.
\end{abstract}

Keywords: radioactivity; decommissioning; BWR; ORIGEN; DORT; TORT

\section{Introduction}

Accurate estimation of radioactivity for a whole region in a nuclear power plant is getting more and more important for a decommissioning plan, especially in terms of radioactive waste processing and radiation protection plan for workers. It is general to calculate radioactivity at local points in a plant with calculation conditions such as elemental composition, neutron irradiation conditions and cooling time at each point using an isotope generation and depletion code such as ORIGEN-S [1]. For a large system with various materials such as a nuclear power plant, the conventional method needs huge amounts of calculations. The number of calculation will rise up to hundreds of thousands for one material because calculations are needed on each mesh point of neutron flux over all regions of interest. The amount of calculations depends on the calculation conditions. Moreover, the change of calculation conditions also needs huge amounts of recalculations.

As one of reasonable methods, calculation is done for each of small sub region of the whole system having certain largeness and representative neutron conditions. However, this method has three technical problems. First, it is not clear how to divide the region and to set the neutron conditions. Second, the application of a conservative flux value to a sub region results in

*Corresponding author. Email: masahiko.kurosawa@toshiba.co.jp overestimation of radioactive waste amount. Third, it is difficult to decide the level boundary of radioactive waste.

According to the above situation, we developed a new method for radioactivity calculation for each point in a shorter time than the conventional method with the same accuracy. The essential point of this method is the application of radioactivity database which is prepared by prior calculation with typical conditions.

\section{Description of the method}

The method has four main steps in order to obtain the radioactivity all over the plant. The processing flow is shown in Figure 1 and explained below from (1) to (4). The important point is after radioactivity calculations. In Figure 1, boxes with sharp corner represent actions and boxes with round corner represent data.

(1) Radioactivity calculation

At the first step, radioactivity of each nuclide is calculated for typical neutron irradiation conditions, cooling times and elemental compositions in a nuclear power plant.

The typical neutron irradiation conditions are neutron irradiation time, irradiation interval, thermal neutron flux $(\mathrm{E}<0.68 \mathrm{eV})$, ratio of the fast neutron $(\mathrm{E}>1 \mathrm{MeV})$ flux relative to thermal neutron flux $(F A S T)$ and the flux of epithermal neutron relative to thermal neutron (RES). Here, these 'FAST' and 'RES' are the same definitions in 
ORIGEN-S. The conditions of the neutron flux are selected based on the result of the neutron flux distribution calculation by two-dimensional discrete ordinates transport code DORT [2] or three-dimensional TORT [3]. The ranges of the neutron flux are set so as to include the range in nuclear power plants of interest.

(2) Radioactivity database preparation

At the second step, the radioactivity database applicable to the whole region of the plant is prepared by repeating and accumulating the results of the first step. The database consists of nuclide production for the typical neutron conditions per unit mass of each material.

(3) Interpolation or extrapolation of the database

At the third step, radioactivity corresponding to a specific neutron condition in each mesh point of the neutron flux calculation by using DORT or TORT are interpolated or extrapolated from the database with a method such as spline interpolation.

(4) Radioactivity assessment

At the forth step, the radioactivity all over the region is accumulated by repeating the third step.

Accordingly, the method can recalculate radioactivity within a shorter time than conventional method with the database based on the typical conditions even when neutron flux and cooling time change.

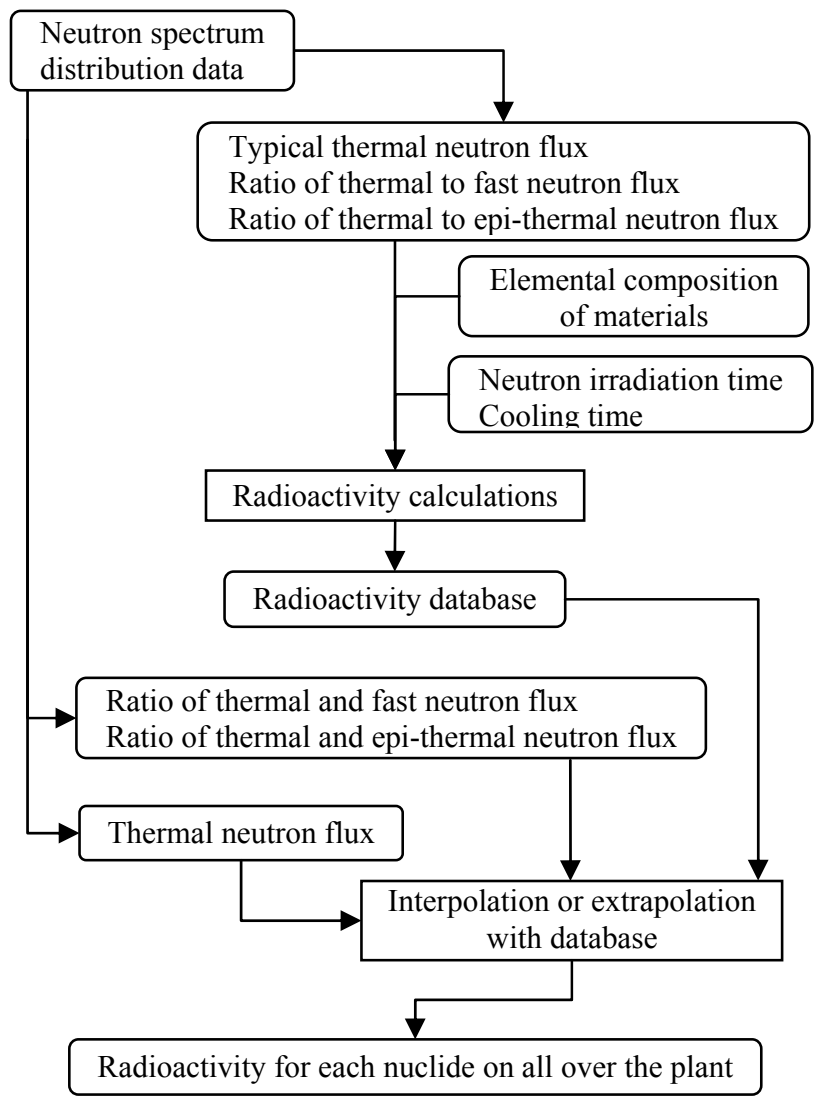

Figure 1. The scheme of the developed method for calculating radioactivity in the nuclear power plant.

\section{Effects of the method application for an existing plant}

Two-dimensional and three-dimensional radioactivity databases are applied to the developed method as Radioactivity database mentioned in Figure 1. The two-dimensional (2D) database expresses a radioactivity database with the parameters of RES and FAST for typical thermal neutron flux. The three-dimensional (3D) database expresses a database with parameters of thermal neutron flux, RES and FAST. The radioactivity for each flux and ratio exists in the database.

The preparation time of $2 \mathrm{D}$ database is shorter than the one of $3 \mathrm{D}$ database. A result with $2 \mathrm{D}$ database may not have good accord with the conventional method in high neutron flux region. It is because the correction factor $n$ which is defined in Section 3.1.1 is set as constant in 2D database for whole flux region. The correction factor $n$ of some nuclides is not constant in high flux region because of the extinction of the original nuclide and a main generation process shifts to a different process. Therefore, extension of the database from $2 \mathrm{D}$ to $3 \mathrm{D}$ is carried out over whole flux regions. Radioactivity in a point is directly interpolated or extrapolated with 3D database.

The criterion which database is used depends on the demand for the accuracy and time of radioactivity evaluation. The accuracy and time of the calculation with the database depend on various conditions of each nuclear power plant.

\subsection{Two-dimensional database application}

\subsubsection{Database preparation}

Consideration of specific conditions is very effective in order to apply the method more reasonably. Figure 2 shows logarithmic plots for radioactivity production of ${ }^{60} \mathrm{Co},{ }^{32} \mathrm{Si},{ }^{137} \mathrm{Cs}$ and ${ }^{242} \mathrm{Pu}$ obtained using ORIGEN-S for thermal neutron flux with consideration of irradiation and cooling time. The four nuclides are selected in order to clarify the effectiveness of the method because the characteristics in the figure are respectively different. In this figure, the productions are simply proportional to the thermal neutron flux for ${ }^{60} \mathrm{Co}$ and ${ }^{137} \mathrm{Cs}$, a quadratic function for ${ }^{32} \mathrm{Si}$ and a mixed function of quadratic and another of the thermal neutron flux for ${ }^{242} \mathrm{Pu}$. So if the radioactivity database is prepared for one typical thermal neuron flux with various FAST and RES values, the radioactivity for the neutron conditions obtained by DORT or TORT is assumed as the following;

$$
C=C F \times\left(\frac{\Phi}{\Phi_{\text {base }}}\right)^{n}
$$

Here, $C$ is the radioactivity in a point. $\Phi$ is the thermal neutron flux at the mesh point. $C F$ is the radioactivity obtained from the database for the FAST and RES values at the mesh point in the neutron flux distribution because the database is prepared as a two dimensional map with typical FAST and RES for the typical neutron flux $\Phi_{\text {base }}$. The effect of the difference of the thermal neutron flux between $\Phi_{\text {base }}$ and $\Phi$ should be corrected considering the 
radioactivity production characteristic shown in Figure 2. The correction factors $n$ in above equation might be $n=1$ for ${ }^{60} \mathrm{Co}$ and ${ }^{137} \mathrm{Cs}, n=2$ for ${ }^{32} \mathrm{Si}$. No appropriate value is for ${ }^{242} \mathrm{Pu}$ because the function for ${ }^{242} \mathrm{Pu}$ is neither quadratic nor another. The value might be 2 or another because the correction factor must be just one value in 2D database although calculation accuracy would become worse.

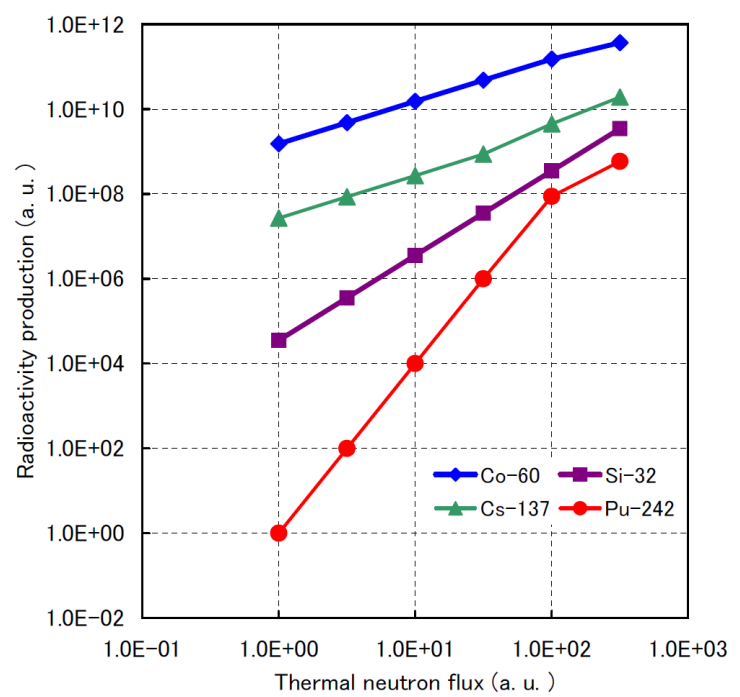

Figure 2. Radioactive nuclides productions for typical thermal neutron flux.

\subsubsection{Application to existing BWR}

The developed method was applied to a BWR with power around $400 \mathrm{MWe}$ in Japan. The neutron flux distribution was calculated by DORT with the nuclear data library MATXSLIB-J33 [4]. The calculation conditions are 175 neutron energy groups, $\mathrm{P}_{5}$ as an order of scattering expansion and $\mathrm{S}_{12}$ of number of directions. The number of mesh points in this calculation was around 400,000. The neutron distribution calculated by DORT extends to outer of the biological shielding wall (BSW) in radial direction.

The database was prepared with the method described in the previous section. The correction factors $n$ were assumed to be constant numbers for about 120 radioactive nuclides. Covering the ranges of ' $F A S T$ ' and ' $R E S$ ' with $10^{10} \mathrm{n} / \mathrm{s} \cdot \mathrm{cm}^{2}$ thermal neutron flux at each mesh point, the number of calculation cases was about 120 for each radioactive nuclide produced.

Figure 3 shows an example of using the method for ${ }^{137} \mathrm{Cs}$ together with the results of using ORIGEN-S. The result was obtained from the inner of the reactor shielding wall (RSW) to the outer of BSW at the height of the core center. Figure 3 shows the result of the developed method on ${ }^{137} \mathrm{Cs}$ is in good accord with the conventional method in the region. Therefore, the developed method is applicable to radioactivity estimation because the described region is important for decommissioning from the points of radioactive waste amount and the boundary for radioactive waste disposal level.

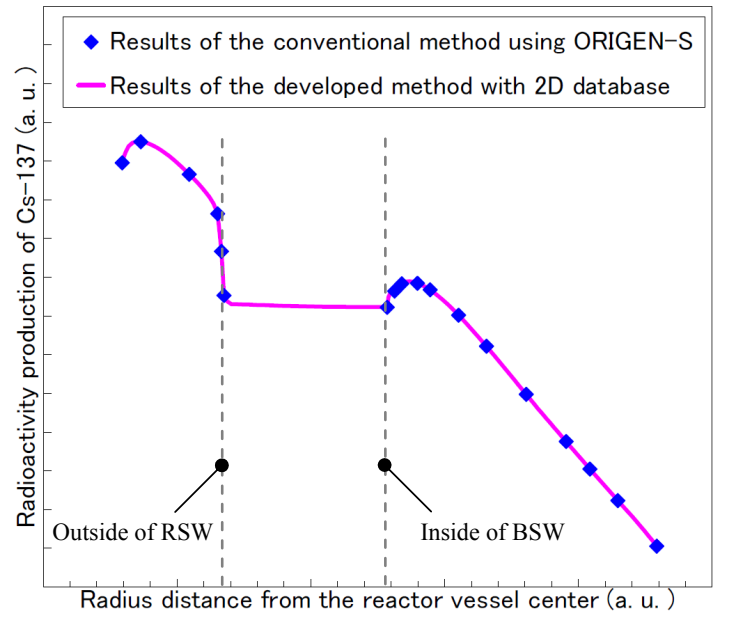

Figure 3. Results of the ${ }^{137} \mathrm{Cs}$ radioactivity production calculation by the developed method with $2 \mathrm{D}$ database together with the conventional method using ORIGEN-S.

\subsection{Three-dimensional database application}

\subsubsection{Database preparation}

In Figure 2, the relationships between the neutron flux and production of some nuclides are not constant over the whole range of the neutron flux. The relationship is set as constant in 2D database and it may not result in good accuracy. Extensions of $n$ and database were considered as solutions. The extension of $n$ means that $n$ is designed to be a function of thermal neutron flux, however, it complicates the way of interpolation or extrapolation. Instead of the above solution, the database is extended to include thermal neutron flux. Interpolation or extrapolation is very simple in this extended (3D) database although this method results in an increase of database preparation time.

\subsubsection{Application to existing BWR}

The developed method with 3D database was applied to above-mentioned BWR plant. Figure 4 shows results of ${ }^{137} \mathrm{Cs}$ production by the developed method in comparison with those by 2D database and ORIGEN-S.

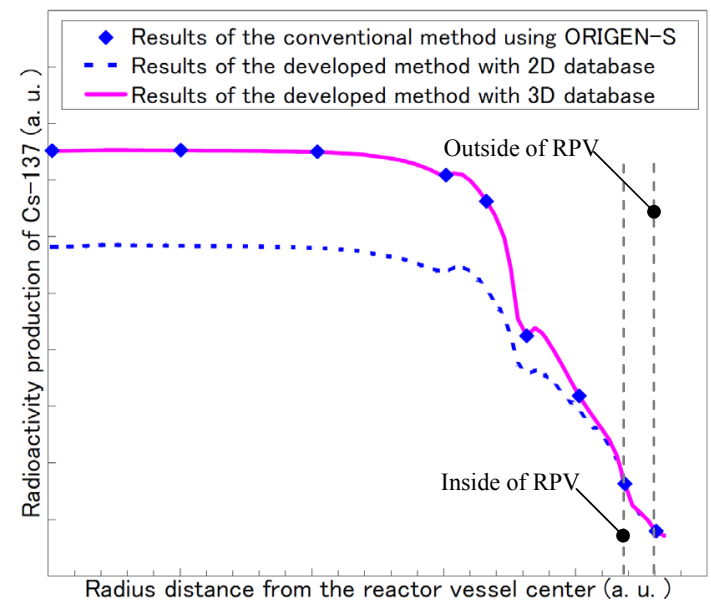

Figure 4. Results for ${ }^{137} \mathrm{Cs}$ radioactivity production calculation by the conventional method using ORIGEN-S and the developed method with 2D and 3D database. 
The results with $3 \mathrm{D}$ database are in good accord with the conventional method using ORIGEN-S in the core region and the reactor pressure vessel (RPV) which have high neutron flux.

Figure 5 shows a radioactivity distribution map for ${ }^{137} \mathrm{Cs}$. A detailed boundary for radioactive disposal level can be derived from the map.

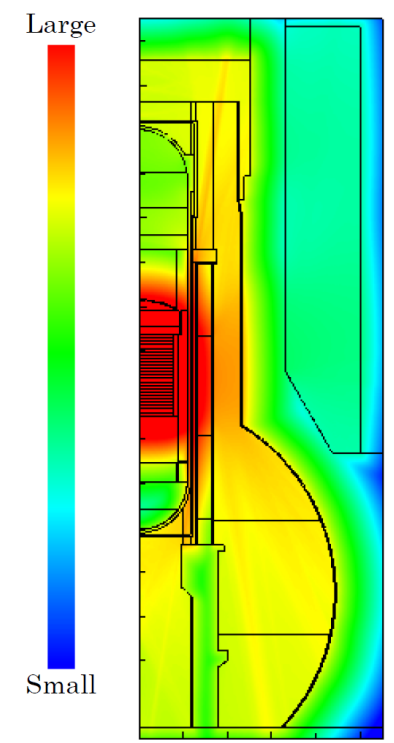

Figure 5. The radioactivity production map of ${ }^{137} \mathrm{Cs}$ to the outer of the biological shielding wall just after a reactor shutdown.

\subsection{Performance of the developed method}

Calculation time is 400,000 minutes in the conventional method with ORGEIN-S, 100 minutes in the developed method with 2D database and 600 minutes in the developed method with 3D database under the evaluation condition of 1 minute for 1 ORIGEN-S calculation and 400,000 mesh points in a plant. The developed method can shorten the calculation time up to around 1/4000 without loss of accuracy. The result of 3D database application was identical with the conventional method in around $99 \%$ cases. Moreover, the radioactivity database can be used repeatedly even when the conditions change while whole calculations must be repeated in the conventional method.

The application of the developed method to the radioactivity estimation in nuclear power plants has the following advantages.

(1) The calculation time is shortened to around $1 / 4000$.

(2) The accuracy is almost same with the conventional method.

(3) The radioactivity in each point of the plant is calculated with reasonable safety margin.

The method can immediately evaluate the radioactive level boundary and total amount of wastes disposal with good accuracy. Thus, it can be applied to the permission and authorization relative to the radioactive wastes evaluation.

In the developed method, the type of the database applied to radioactivity calculation should be selected with consideration of accuracy and calculation time because a result with $2 \mathrm{D}$ database may not have good accord with the conventional method for some nuclides in high neutron flux region as mentioned in Section 3.

\section{Conclusion}

The number of the plant with long operation term has increased in the world. Therefore, a method which enables radioactivity evaluation in a short time and in the same accuracy with the conventional method is very important to determine the level boundary of radioactive waste.

A method was developed as the solution. It uses radioactivity database effectively and does not require detail calculations in each mesh point. It enables to calculate radioactivity in a shorter time than the conventional method with the same accuracy.

Development will be promoted through further verification and validation on wider application to the existing plants. For example, the extensions of the object nuclides and the nuclide generation process for radioactive waste disposal are necessary.

\section{Acknowledgements}

The authors wish to thank Hidenori TANABE, Hideaki ICHIGE of The Japan Atomic Power Company for the helpful support to this work.

\section{References}

[1] L. C. Gauld, O. W. Herman and R. M. Westfall, ORIGEN-S: SCALE System Module to Calculate Fuel Depletion, Actinide Transmutation, Fission Product Buildup and Decay, and Associated Radiation Source Terms, ORNL/TM-2005/39, Oak Ridge National Laboratory, (2006).

[2] J. O. Johonson, A User's Manual for MASH 1.0 - A Monte Carlo Adjoint Shielding Code System, ORNL/TM-11778, Oak Ridge National Laboratory, (1992).

[3] W. A. Rhoades and D. B. Simpson, The TORT Three-Dimensional Discrete Ordinates Neutron/Photon Transport Code, ORNL/TM-13221, Oak Ridge National Laboratory, (1997).

[4] K. Kosako, N. Yamano, T. Fukahori, K. Shibata and A. Hasegawa, The Libraries FXLIB and MATXSLIB based on JENDL-3, JAERI-Data/Code 2003-011, Japan Atomic Energy Research Institute, (2003). 